\title{
Emergence of chikungunya in Moonlapamok and Khong Districts, Champassak Province, the Lao People's Democratic Republic, May to September 2012
}

\author{
Chanthavy Soulaphy, ${ }^{a}$ Phouthone Souliphone, ab Khonesavanh Phanthavong, bc Darouny Phonekeo, \\ Sonesavanh Phimmasine, ${ }^{d}$ Bouaphan Khamphaphongphane, ${ }^{a}$ Viengsavanh Kitthiphong ${ }^{e}$ and Hannah C Lewis ${ }^{d}$ \\ Correspondence to Chanthavy Soulaphy (e-mail: chanthavyncle@hotmail.com).
}

Introduction: Chikungunya is a vector-borne disease transmitted to humans by Aedes mosquitoes, which are widespread in the Lao People's Democratic Republic. However, chikungunya virus (CHIKV) had not been detected in the country before outbreaks reported in July 2012. The first outbreaks were detected through health care worker event-based surveillance.

Methods: The case definition for the outbreaks was defined as a person with acute onset of fever $\left(\geq 38{ }^{\circ} \mathrm{C}\right)$ and severe arthralgia (joint pain) or arthritis from 1 May 2012 in Champassak Province. Rapid response teams conducted active case finding, performed an environmental assessment including an entomological survey and implemented control measures. Descriptive analysis was undertaken in Microsoft Excel.

Results: There were 197 cases (attack rate 3.4\%) of suspected chikungunya reported from 10 villages in Moonlapamok and Khong Districts of Champassak Province. All age groups (age range: seven months-74 years) were affected with slightly more female (56\%) than male cases. Thirty-one per cent (16 of 52) of serum samples tested positive for CHIKV by polymerase chain reaction. The environmental assessment found poor water storage practices and high entomological indices.

Discussion: These outbreaks show the effectiveness of health care worker event-based surveillance and the importance of sharing of information across borders for detecting emerging diseases. Public health education is an important measure to prevent epidemics of chikungunya. Information about chikungunya should be supplied to health care workers in the region so they are alert to the potential spread and are able to implement control measures for this disease.

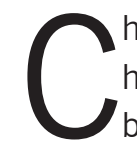
hikungunya is a vector-borne disease transmitted to humans by Aedes mosquitoes and is characterized by sudden onset of high fever and severe joint pains. Although rarely fatal, chronic conditions such as rheumatic symptoms and depression are common and debilitating. ${ }^{1}$ Many parts of Asia are considered endemic for chikungunya. The Lao People's Democratic Republic is a land-locked country in South-East Asia that borders Cambodia, Thailand, Viet Nam, China and Myanmar. Although the Aedes mosquitoes vectors are widespread in the Lao People's Democratic Republic, ${ }^{2}$ Chikungunya virus (CHIKV) had not been detected in the country before July 2012.

On 13 July 2012, surveillance staff from the National Centre for Laboratory and Epidemiology (NCLE) were conducting routine work in Champassak Province in the far south of the Lao People's Democratic Republic. They were informed by Moonlapamok District health care workers about patients with fever, rash, body pain and joint pain in Thakang village, a remote village $8 \mathrm{~km}$ from the Cambodian border. As there had been recent

\footnotetext{
- National Center for Laboratory and Epidemiology, Ministry of Health, Vientiane, Lao People's Democratic Republic. Lao Field Epidemiology Training, Ministry of Health, Lao People's Democratic Republic.

Centre for Malaria, Parasitology and Entomology, Ministry of Health, Vientiane, Lao People's Democratic Republic. World Health Organization Country Office, Vientiane, Lao People's Democratic Republic.

Department of Communicable Disease Control, Ministry of Health, Vientiane, Lao People's Democratic Republic.

Submitted: 15 November 2012; Published: 18 March 2013

doi: 10.5365/wpsar.2012.3.4.017
} 
Figure 1. Epidemic curve of chikungunya cases $(n=197)$ in Moonlapamok and Khong Districts, Champassak Province, May to September 2012

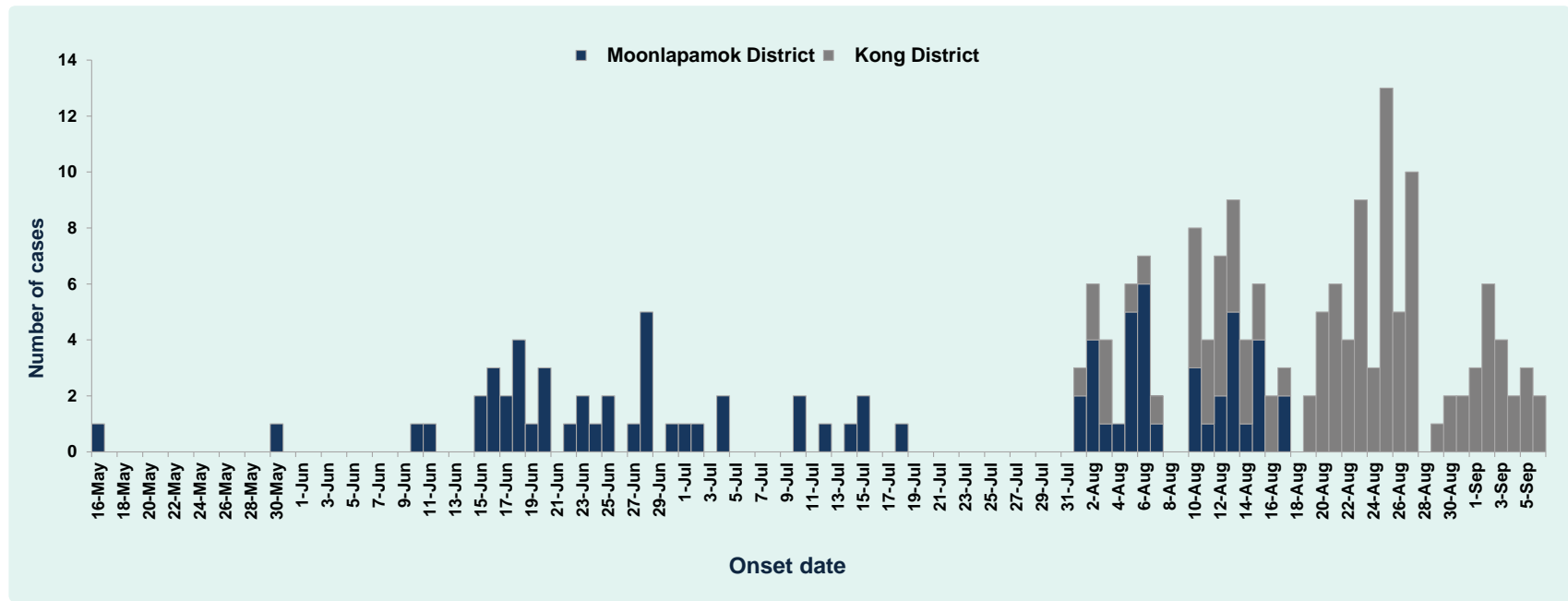

outbreaks of chikungunya reported in Cambodia, one of the NCLE surveillance staff thought these cases could possibly be chikungunya.

In response, an outbreak investigation was conducted by district and provincial authorities. In the same month, two further outbreaks of suspected chikungunya were reported from two new villages in Moonlapamok District - Kaanleuag and Nadi. With the spread of this emerging disease, the NCLE deployed a central Rapid Response Team (RRT). Between July and September 2012 further villages in both Moonlapamok and Khong Districts reported outbreaks of suspected chikungunya. We describe the investigations and control measures established for these outbreaks and discuss recommendations for chikungunya control in the Lao People's Democratic Republic.

\section{METHODS}

District and provincial RRTs were deployed for the first two reported outbreaks in Thakang and Kaanleuag villages, Moonlapamok District. A central RRT comprised of staff from the NCLE, field epidemiology trainees from the Department of Disease Control and the World Health Organization joined provincial and district response teams to investigate the outbreak in Nadi villages, Moonlapamok District, Champassak Province from 7 to 11 August 2012. Outbreaks in four further villages (Doneheid, Donekhao, Hangsadam, Vernkham) in Khong District were investigated by district and provincial RRTs from August to September 2012. The results of these investigations were sent to NCLE for analysis.

The teams conducted active case-finding by interviewing village leaders and village health volunteers in affected villages and doing a retrospective review of logbooks in the Champassak provincial hospital, affected district hospitals and affected village health centres. Active case finding was also conducted in five villages neighbouring Nadi village, Moonlapamok District by the central RRT.

The case definition for all outbreaks was defined as a person with acute onset of fever $\left(>38^{\circ} \mathrm{C}\right)$ and severe arthralgia (joint pain) or arthritis since 1 May 2012 in Champassak Province. All cases meeting the case definition were recorded in a line list (name, age, location, onset date, symptom, sample collection). For cases meeting the case definition, with onset in the previous five days, serum were samples collected and tested for CHIKV by polymerase chain reaction (PCR) at NCLE. As there were positive samples for CHIKV, testing was not done for other pathogens, as per the testing strategy.

An environmental assessment including a larva survey was carried out by the central RRT in 35 households of Nadi village, Moonlapamok District. House Index (HI: percentage of houses positive for larvae), Container Index ( $\mathrm{Cl}$ : percentage of waterholding containers positive for larvae) and Bretaeu Index 
Figure 2. Distribution of chikungunya outbreaks in Moonlapamok and Khong Districts, Champassak Province, July to September 2012

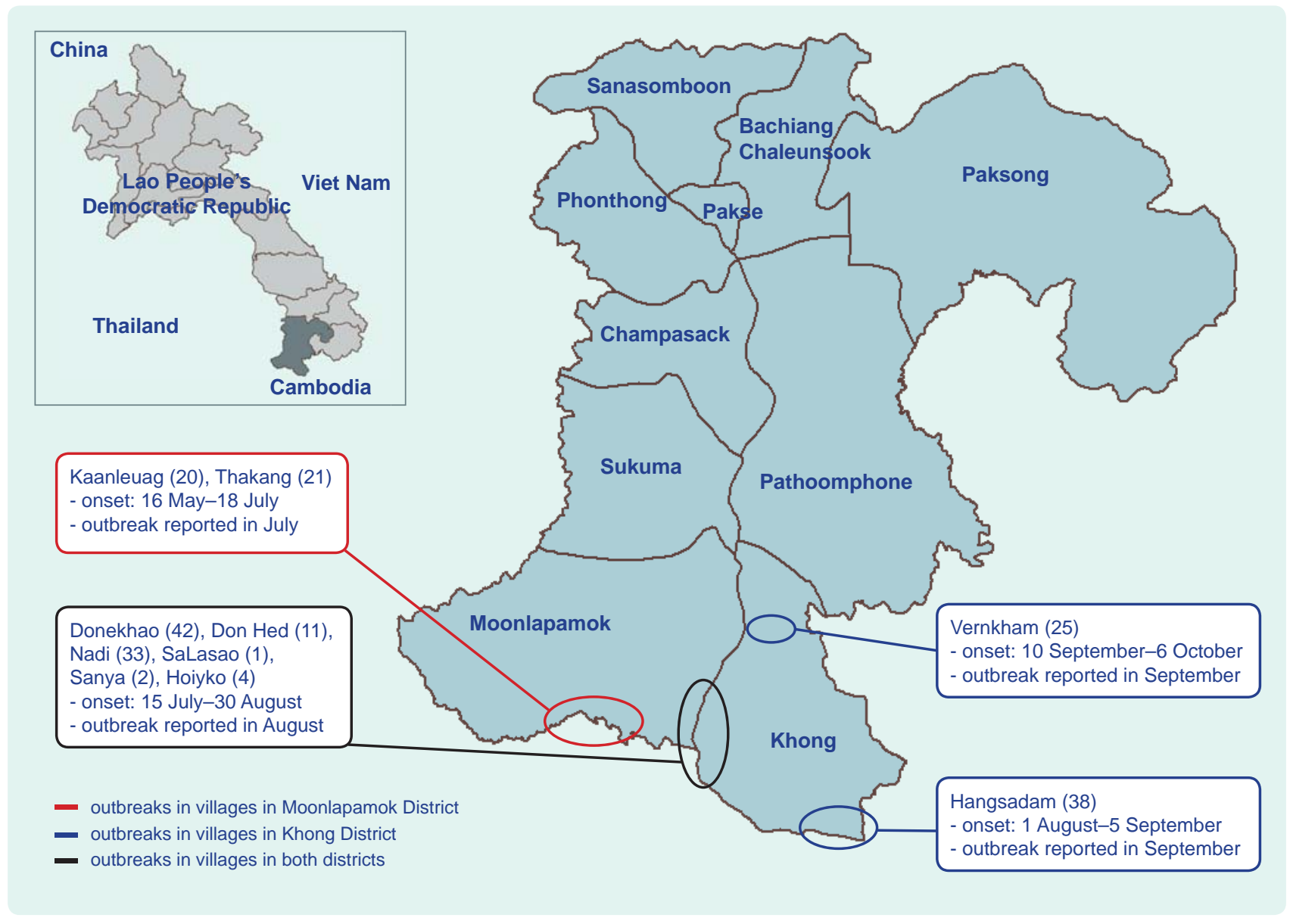

Note: Numbers in parenthesis refer to suspected cases.

(BI: number of positive containers per 100 houses) were calculated. Descriptive analysis was undertaken in Microsoft Excel.

\section{RESULTS}

Between 1 May and 6 September 2012, 197 cases of suspected chikungunya were reported from 10 villages in Moonlapamok and Khong Districts of Champassak Province with onset between 16 May and 6 September (Figures 1 and 2).

The overall attack rate was 3.4\% (197/5807) but was higher in certain villages such as Donkhao (42/277, $15 \%)$ in Khong District and Kanleung (20/187, $11 \%$ ) in Moonlapamok District (Figures 2). All age groups were affected, and ages ranged from seven months to 96 years old (median: 35 years; interquartile range: 50-16 $=34$ years). More females $(122 / 197,62 \%)$ than males were affected but the attack rate was slightly higher in males $(2.8 \%)$ than females $(2.1 \%)$.

As per the case definition, all cases had fever and all had arthralgia. Other clinical signs and symptoms reported were petechia rash (82\%) and body pain (73\%). A total of 52 serums samples were tested and $16(30.8 \%)$ were positive for CHIKV.

All cases were reported from health centres and villages and no cases were detected from district or provincial hospital logbook records. Some cases with similar symptoms were diagnosed as rheumatic fever and were followed up but not thought to be associated with the outbreaks.

The environmental survey found water from the Khong River was being stored in large jars outside the majority of homes; the jars were not changed regularly or 
covered. For the entomological survey of 35 households, the following indices were estimated: $\mathrm{HI}: 51 \%, \mathrm{Cl}: 24 \%$ and $\mathrm{BI}: 85$.

The villagers were provided health education on community clean-up to prevent mosquito breeding sites such as rubbish and water containers. Villagers were also advised to avoid mosquito bites and sleep under mosquito nets, especially if symptomatic, to prevent onward transmission. Villagers were also advised to visit a health care worker if they became sick. Provincial and district health workers were provided training on CHIKV epidemiology and control measures and informed to report any outbreaks immediately.

The last reported case of suspected chikungunya was on 6 September 2012 in Vernkham village, Khong District.

\section{DISCUSSION}

Chikungunya is an emerging disease in South-East Asia. Sporadic cases of chikungunya have been reported in Cambodia, including in provinces bordering the Lao People's Democratic Republic, since 2011, and recent outbreaks affected rural communities in early 2012.3,4 The Lao People's Democratic Republic is a landlocked country bordered by Thailand, Viet Nam, China, Myanmar and Cambodia. In Thailand, the first appearance of chikungunya was in 1960, and many outbreaks have been reported over the years since then. ${ }^{5}$ China experienced its first outbreaks in $2010 .{ }^{6}$ Other countries in the region such as India, Sri Lanka and Malaysia have also reported outbreaks. ${ }^{7-9}$ Between July and September 2010, the Lao People's Democratic Republic conducted the first chikungunya sero-survey in Vientiane Capital with all nine district and six central hospitals. Two hundred serum samples were tested by enzyme-linked immunosorbent assay (ELISA) and PCR for Chikungunya. However, no samples were positive for CHIKV (unpublished data, NCLE). Both adults and children were affected by this outbreak meaning that adults were not immune and that the disease was new to this area. Therefore, we believe these outbreaks to be the first chikungunya diagnosis in the Lao People's Democratic Republic and therefore that chikungunya is emerging in the Lao People's Democratic Republic.
Between May to September 2012, 195 suspected chikungunya cases were reported to NCLE and investigated. This is a low number of cases compared to other countries in the region. There were some limitations to these outbreak investigations which could lead to underestimation or overestimation of the number of cases. First, cases with milder symptoms were not included in the outbreak case definition and so the number of cases presented here likely underestimates the size of the outbreak. Furthermore, cases may have gone undetected due to misdiagnoses as other febrile diseases with similar clinical presentations to chikungunya (e.g. dengue, malaria and measles). On the other hand, only a small number of the cases were laboratoryconfirmed, so other infections may be responsible for some of the clinically suspected chikungunya cases. Of note, there were cases of malaria and dengue confirmed in the affected districts during the period of the outbreak (May to September), and dengue virus shares the same vector. These diseases cannot be excluded and were not tested for due to limited reagent availability.

This emerging disease must continue to be taken seriously, and prevention and control measures should be established or strengthened in affected areas. Public health education about chikungunya, such as etiology and incidence of the disease, disease prevention, vector mosquitoes and their control should be distributed to the public and supplied to health care workers so they are alert to the potential spread and emergence of this disease. As the vectors are the same as for dengue, this information could be supplied together for both diseases. Personal protection from biting mosquitoes is also a critical measure that could minimize the expansion of chikungunya in epidemic areas. Advice on the use of mosquito nets (including when sleeping during the daytime) and the use of mosquito repellents and mosquito coils should also be encouraged.

This first report of chikungunya in the Lao People's Democratic Republic was made by a surveillance officer who had learnt of outbreaks in Cambodia. All further outbreaks were also detected and reported via health care workers to the Lao People's Democratic Republic event-based surveillance system. This provides good evidence of the effectiveness of health care worker event-based 
surveillance and cross-border information sharing in detecting new emerging diseases and responding to them appropriately.

\section{Conflicts of interest}

None declared.

\section{Funding}

None.

\section{References:}

1. Preparedness and Response for Chikungunya Virus. Introduction to the Americas. Washington DC, Pan American Health Organization and Centers for Disease Control and Prevention, 2011.

2. Tsuda $Y$ et al. An ecological survey of dengue vector mosquitos in central Lao PDR. Southeast Asian Journal of Tropical Medicine and Public Health, 2002, 33:63-67. pmid:12118462
3. Centers for Disease Control and Prevention (CDC). Chikungunya outbreak-Cambodia, February-March 2012. MMWR. Morbidity and Mortality Weekly Report, 2012, 61:737-740. pmid:22992571

4. Duong $V$ et al. Reemergence of Chikungunya virus in Cambodia. Emerging Infectious Diseases, 2012, 18(12):2066-2069. doi:10.3201/eid1812.120471 pmid:23171736

5. Thaikruea $L$ et al. Chikungunya in Thailand: a re-emerging disease? Southeast Asian Journal of Tropical Medicine and Public Health, 1997, 28:359-364. pmid:9444021

6. Qiaoli Z et al. Maiden outbreak of chikungunya in Dongguan city, Guangdong province, China: epidemiological characteristics. PLOS ONE, 2012, 7:e42830. doi:10.1371/journal. pone. 0042830 pmid:22916166

7. Hapuarachchi $\mathrm{HC}$ et al. Re-emergence of Chikungunya virus in South-east Asia: virological evidence from Sri Lanka and Singapore. Journal of General Virology, 2010, 91:1067-1076. doi:10.1099/vir.0.015743-0 pmid:19955565

8. Rozilawati $\mathrm{H}$ et al. Entomological study of chikungunya infections in the State of Kelantan, Malaysia. Indian Journal of Medical Research, 2011, 133:670-673. pmid:21727669

9. Tandale BV et al. Systemic involvements and fatalities during Chikungunya epidemic in India, 2006. Journal of Clinical Virology, 2009, 46:145-149. doi:10.1016/j.jcv.2009.06.027 pmid: 19640780 